\title{
Comparison of safety and kinematic patterns of automated vehicles turning left in interaction with oncoming manually driven vehicles
}

\author{
MAREK JUNGHANS ${ }^{a}$, FLORIAN KRAUNS ${ }^{b}$, ADRIAN SONKA ${ }^{c}$, MICHAEL BÖHM ${ }^{a}$, MANDY DOTZAUER \\ a. German Aerospace Center (DLR), Institute of Transportation Systems, Rutherfordstraße 2, 12489 Berlin, Germany \\ b. The work described was conducted at Technische Universität Braunschweig, Institute of Automotive Engineering, \\ F. Krauns is now employed by Volkswagen Commercial Vehicles, Volkswagen AG, Mailbox 1515, 38436 Wolfsburg, Germany \\ c. Technische Universität Braunschweig, Institute of Automotive Engineering, Hans-Sommer-Straße 4, 38106 Braunschweig, \\ Germany
}

KEYWORDS: automated driving; traffic safety; road user behaviour; conditionally tolerable left turn, trajectory data analysis

ABSTRACT: Highly and fully automated driving has been under development for the past two decades in order to increase comfort, efficiency, and traffic safety. Particularly in the latter domain, experts agree on automated driving, especially in case of automated vehicles (AV) with SAE level 4 or higher, having the most promising effects. Automated driving is expected to decrease the number of seriously injured or even killed road users to zero (Vision Zero). However, automated driving is still in an early stage of development and many AV tend to drive very carefully to avoid crashes. So, the goal is to make driving more efficient while maintaining the highest level of safety. In the project "Digitaler Knoten $4.0^{\prime \prime}$ cooperative automated driving was assessed regarding efficiency and safety aspects. One of the use cases investigated was turning left with oncoming traffic at an urban intersection as this situation represents one of the most complex situations in urban areas yielding to crashes with-in many cases-serious consequences for the involved road users. At the Application Platform Intelligent Mobility (AIM) Research Intersection in Braunschweig, Germany, an SAE level 3 AV was turning left interacting with oncoming manually driven vehicles (MV). The performance of the AV was compared to MV executing the same manoeuvre. The recorded video-based trajectories of the respective AV as well as MV were analysed regarding the influence of situational factors (e.g. position of the vehicle in the queue and gap acceptance) and kinematic factors (e.g. speed and acceleration) on traffic safety. The similarities and differences between this specific AV and MV were identified yielding insight for further developing algorithms for more efficient driving while maintaining the same traffic safety level. For instance, it appears that the AV shows a very conservative left turning behaviour leading to very safe PET distributions in comparison to left turning MV.

\section{INTRODUCTION}

Although automated driving has become more and more realistic in relatively simple traffic environments (e.g. on motorways), it has not been assured to reduce the number of less severe crashes in urban areas in comparison to manual driving (e.g. NHTSA, 2017; Pink et al., 2015). Also, in the recent past, several fatal crashes with self-driving cars took place (e.g. Fleming, 2016; Green, 2018; Wakabayashi, 2018). This, however, intensified the discussion about the technical degree of maturity of automated driving functions (ADF) and requires the research work ahead. The reason for this is that urban areas are specifically complex and thus one of the greatest challenges for ADF as different road users (motorists, bicyclists, pedestrians, e-scooter drivers, etc.) with different intentions (e.g. commuting, shopping, or leisure time) and different speed meet each other in different traffic areas with different signalling (e.g. traffic lights and traffic signs). Therefore, self-driving cars are equipped with a variety of sensors and assessment algorithms in order to interpret and predict the behaviour of all the surrounding road users in the near future. Here, comprehensive and powerful artificial intelligence (AI) methods take effect. But due to a large gap of required training data, an AI method needs to predict any thinkable situation, making this a challenging undertaking. Brunner et al. (2019) state that this requires more than a billion test kilometres, which is economically unacceptable in a realistic time period. This is one of the reasons self-driving cars currently struggle to avoid crashes.

When different road users with different mobility intentions meet in the same space and time, they interact with each other. These interactions (i.e. a close spatio-temporal relationship between motorised road users and between motorised and non-motorised road users) are based on rules described in the road traffic regulations defining, for example, rules on right of way, overtaking, parking, and turning right or left. However, when it comes to interactions between different road users with different needs, the human capability of anticipating traffic situations mostly leads to non-critical and controlled, but sometimes also critical encounters with a higher probability of a collision or a severe crash. In case of conflicts, an evasive action of at least one of the interacting road users has to be taken to avoid the crash (e.g. Hydén, 1987; Ismail et al., 2011; Tarko, 2019). In order to fulfil the requirements to reduce the number and severity of crashes by bringing automated vehicles (AV) on the roads, eventually, ADF need to have a sense of "self" (e.g. Jenssen et al., 2019) to understand, anticipate, and predict different road user behaviours in complex and sometimes arbitrary situations in order to plan, implement, communicate, and-if necessary-correct a planned manoeuvre in real-time. Reasons contributing to such critical situations are manifold, for instance, deficits in the transportation infrastructure (e.g. poor road conditions, incomprehensibly complex intersections, and number 
of traffic signs), ambiguous traffic control (e.g. conditional tolerable left turns), errors of road users, unfavourable environmental conditions (e.g. weather, illumination, and visual conditions) and the "state" and situation awareness of road users (e.g. distraction from secondary tasks or mental conditions). Particularly for ADF in urban areas, it is important to not only assure safety and maintain an efficient traffic flow, but to understand interaction behaviour between motorised road users and between motorised and non-motorised road users. This can lead to sensible maturing of ADF "behaving" like humans while being safer (and more efficient).

Therefore, the objective of the study is to investigate the differences between AV and MV turning left with oncoming traffic based on real interaction data. At an urban intersection, video-based trajectory data was recorded and the performance of the AV was compared to MV executing the same manoeuvre.

\section{RELEVANT WORK, RESEARCH QUESTION AND HYPOTHESES}

\subsection{Automated driving functions (ADF)}

Several public and commercial R\&D projects on automated driving were conducted within the last two decades. For instance, robotics competitions such as the DARPA Urban Challenge were firstly examined in artificial environments enabling interactions between AV and MV (e.g. Urmson et al., 2009). Since then, ADF were developed and tested in real traffic environments with safety drivers, for instance, in the German project Stadtpilot (Wille et al., 2010) or the Bertha Benz Drive for which Daimler reconstructed the first cross-country automobile journey of 1888 with an AV (Ziegler et al., 2014).

However, currently, vehicles with SAE level 3 or higher are not mass-produced for urban environments. Car manufacturers just began implementing autonomous emergency braking as SAE level 1 driver assistance system for left and right turn scenarios driven by increasing safety demands of the European NCAP roadmap (see EURO NCAP, 2017 and 2019) and legal requirements such as the European regulations on type-approval requirements for motor vehicles (see EU Regulation, 2019). In recent years, car manufacturers such as Tesla and Volkswagen, but also internet-related technology companies such as Waymo and Uber developed automated urban robotaxis for commercial use (e.g. Berman, 2020; Graber et al, 2019; Ohnsman, 2020), but their technical realisation is confidential in most cases. Only a few insights are given (e.g. Fairfield et al., 2014). In this patent, the conditionally tolerable left turn with oncoming traffic was chosen as an example for a very demanding task for the automated driving function (ADF) in which human as sistance may be needed.

The state of the art of ADF can be considered in different ways. The following examples give a brief insight in what has been done so far to make automated driving possible, particularly the entities relevant for the development of the ADF of the vehicle TEASY 3 (Testing and Engineering of Automated Driving SYstems), used for realising this study (see section 3.2.2). These are: system architecture, road user detection and prediction, trajectory planning, and lateral/longitudinal control. From an architectural point of view, the ADF can be divided into the layers navigation, guidance, and stabilization (e.g. Matthaei et al., 2015) requiring structural modules of the ADF in terms of self-perception (reliable detection and localisation of the ego-vehicle), environmental perception (reliable detection and localisation of the surrounding traffic), and mission accomplishment. Krauns et al. (2019) proposed the structural modules environment \& context model, decision making \& motion planning, and longitudinal \& lateral control. This proposed architecture allows to interpret the extracted environmental features of the detected road users in the traffic context and to predict the road users' behaviours. For instance, Liebner et al. (2013) and Sonka (2020) proposed approaches for estimating driver intentions and for predicting object movements in intersection scenarios. González et al. (2016) gave a comprehensive overview of various algorithms for motion and trajectory planning. They classified them into the categories graph search, sampling-based planners, interpolation methods, and numerical optimisation. Noh (2019) proposed a decision-making framework for autonomous driving on the basis of digital maps and predicted future paths of the road users determining appropriate manoeuvres for an AV to navigate through an intersection safely and efficiently. The AV identifies potential threats and avoids collisions, and it avoids overly conservative driving regarding safety leading to crashes, delays, and deadlocks (e.g. Zhan et al., 2016). Shu et al. (2020), Hubmann et al. (2018) and Liu et al. (2020) proposed deep learning methods and partially observable Markov decision processes on the trajectory planning and decision processes for AV to overcome the inefficient driving of $\mathrm{AV}$ at signalised and unsignalised intersections considering the unknown intentions of the oncoming traffic in uncertain and noisy environments. Ziegler et al. (2014) showed a behavioural state chart for manoeuvre planning and a method to create a driving corridor based on detailed digital maps in consideration of observed constraints such as static obstacles and objects. In Tigges et al. (2017), a lateral control concept is discussed. An approach for an optimised continuous distance control based on a sliding mode control is presented in Liesner (2017).

\subsection{Interactions between $\mathrm{AV}, \mathrm{MV}$ and other road users}

Not a lot of literature can be found on interactions between $\mathrm{AV}$ and $\mathrm{MV}$ in general and left turning AV with oncoming MV traffic in particular. Wang et al. (2019) analysed 113 road user crashes in terms of crash perpetration of AV in California, USA, between 2014 and 2018. They found that when the ADF was activated and in case the AV was responsible for the crash, fatalities occurred. In these cases, drivers mainly ignored the take-over requests of the ADF. In contrast, the injury level decreased when the ADF was deactivated and the AV was not responsible for the crash. In Dotzauer et al. (2017), a method was developed to compare road user crashes and near-crashes with and without involvement of $\mathrm{AV}$, in California, USA. On the basis of 40 crashes, it was found that AV appeared 4.8 times more often in road user crashes than MV. Although these publications suggest the AV is overrepresented in (fatal) crashes, it turned out drivers misused the $\mathrm{ADF}$ of the vehicles.

Studies were found investigating interactions between vulnerable road users (VRU, such as pedestrians, cyclists, motorcycles, and e-scooter drivers) and AV as well as with left turn interactions among MV on the basis of photo studies, questionnaires, the application of simulators, or virtual reality (e.g. Hagenzieker et al., 2020; Rad et al., 2020; Vlakveld et al., 2020). Zhou et al. (2014) analysed gap acceptance in conditionally tolerable left turn manoeuvres from the major road at unsignalised intersections in dependence on the number of rejected gaps and their duration adopting the generalised estimating equations approach. In this simulation-based study, for instance, in case of only a non-accepted gap, 50\% of the drivers turned left when the gap was about five seconds and decreased further to approximately four seconds as the number of non-accepted gaps increased to 15. Chan (2006) analysed and characterised human driving behaviour in conditionally tolerable left turn situations with oncoming traffic. Based on field observations at several intersections, it was found that the lengths of the signal phases of traffic lights 
had an influence on the distribution of gap acceptance, while speed and traffic volume caused differences in risk-taking behaviour of human drivers. Ragland et al. (2006) analysed gap acceptance for vehicles turning left across oncoming traffic in terms of finding adequate opportunities to initiate left turn manoeuvres. They found that acceptable gaps varied between three and twelve seconds. Bärgmann at al. (2015) analysed conditionally tolerable left turns with regard to the drivers' comfort- and dread-zone boundaries. The authors suggested boundaries helping developers of ADF to tune their ADF in a way that AV behave more like human drivers. It appeared that in case the drivers were in comfortable driving conditions, the PET (i.e. post encroachment time; a surrogate safety measure that describes how close two interacting road users on a collision course miss each other) showed values of $2.26 \mathrm{~s}$, whereas the PET was about $1.50 \mathrm{~s}$ when the drivers were in hurried driving conditions. Seo et al. (2018) developed an algorithm for safe left turns at intersections by the use of V2V communications. This safety algorithm informed drivers about dangerous situations in left turn manoeuvres. Further, a number of current research projects address automated driving and interactions with other road users. For instance, the German project @City AF (see @City, 2020) aims to understand interactions between road users and manually driven vehicles (MV) in urban areas in order to improve and optimise ADF. The interaction and cooperation between motorists and bicyclists in narrow road sections are of specific interest. But so far, no publication has been identified. Also, in the EU project L3Pilot (see L3Pilot, 2020), AV piloting is addressed to enhance, mature, and optimise ADF of selfdriving cars of many European vehicle OEM. In this project, an evaluation of ADF in terms of its driving behaviour as well as in terms of interaction with other road users takes place. In this respect, Trullos et al. (2021) evaluated several SSM in a roundabout in Germany. Analog to @City, observations of interactions between MV were analysed building the basis for the improvement of ADF.

Results found in the literature suggest that recent research addressed interactions between MV and AV, but lacks real world data. Therefore, the purpose of this study is to investigate the interaction between manually and automatically driven vehicles turning left with oncoming traffic based on real interaction data.

\subsection{Research question and hypotheses}

The research question of this paper is: "What are the differences in conditionally tolerable left turns in oncoming traffic between an automated vehicle $(A V)$ and manually driven vehicles $(M V)$ ?". At the Application Platform Intelligent Mobility (AIM) Research Intersection in Braunschweig, Germany, video-based trajectory data was recorded in May/June 2019 to analyse and compare the left turning behaviour of an SAE level 3 AV with manually driven cars. The recorded trajectories of the AV and MV were analysed regarding the influence of situational factors (e.g. position of the vehicle in the queue, and gap acceptance) and kinematic factors (e.g. speed and acceleration) on traffic safety. The following hypotheses H1-H5 arise:

H1. Speed: The AV is expected to approach, pass, and leave the intersection slower than MV. The reason for this is that ADF in urban areas are still under research and thus, ADF developers maintain a maximum level of safety to avoid crashes. However, in case a crash is unavoidable the AV should release less kinetic energy.

H2. Acceleration: In line with hypothesis $\mathrm{H} 1$, the AV is expected to accelerate and brake less intensive than MV when approaching the common conflict point. This assumption is supported by the fact that there is only one AV available for this study, whereas there are several different human drivers and driving behaviours using different vehicle types.

H3. Distance and time needed to arrive at the conflict point: In line with the hypotheses $\mathrm{H} 1$ and $\mathrm{H} 2$, the AV is expected to brake earlier before the conflict point than MV. Due to approaching the intersection more slowly than MV the AV is expected to have more time and a larger distance to reach the common conflict point.

H4. PET distribution: Due to its driving at a maximum safety level, the AV is expected to have less critical, but instead more uncritical PET values. Therefore, the PET distribution of the AV will be "shifted" to higher PET values than the PET distribution of MV.

H5. Accepted/non-accepted time gaps: In line with the hypotheses $\mathrm{H} 3$ and $\mathrm{H} 4$ the $\mathrm{AV}$ is expected to accept larger gaps intersecting the oncoming traffic than MV.

\section{METHOD}

\subsection{Data}

During May 14 to June 6, 2019, between 11:24 am and 6:08 pm data were collected. Altogether, 39 left turn situations of AV were recorded. Three situations were discarded due to corrupted trajectories and another nine situations due to the fact that the AV did not interact with vehicles of the oncoming traffic. In case of MV turning left, 72 baseline situations (in order to have a 1:2 relation for AV vs. MV) were selected from the whole data set \pm ten minutes around AV situations (i.e. conditions between $\mathrm{AV}$ and MV were comparable). Two situations were discarded as MV did not interact with oncoming traffic. 27 left turns of the AV and 70 left turns of an MV were used for the analysis.

\subsection{Material and apparatus}

\subsubsection{AIM Research Intersection}

The data acquisition took place at the Application Platform Intelligent Mobility (AIM) Research Intersection: An urban intersection at Hagenring/Rebenring in Braunschweig, Germany. The inner part of this intersection was equipped with video-based sensors to detect, discriminate and track road users. In Figure 1 (left) its schematic sensor equipment is shown. Road user trajectories were generated by fusing the different camera sensors in real-time (see a trajectory sample of one hour in the middle of Figure 1). The resulting data were provided in $25 \mathrm{fps}$ and contained information about the time stamps of the road users, their positions, speeds, accelerations, headings, sizes and modes of transport (cars, trucks, vans, motorcycles, pedestrians, bicyclists). The high-resolution video data provided by the cameras were anonymised in real-time to very low-resolution images to fulfil the GDPR restrictions (see augmented video scene in Figure 1 (right)). Vehicle number plates as well as faces of pedestrians and bicyclists could not be detected or tracked. Additionally, the AIM Research Intersection was equipped with infrastructureto-X (I2X) communication modules, called road side units, in order to transmit messages (e.g. signal phase and timing extended message (SPATem); and MAPem ${ }^{1}$ ) to road users, and also receive messages from V2X-communicable vehicles or infrastructure-based devices.

\subsubsection{Automated vehicle TEASY 3}

For performing fully automated left turning manoeuvres in an urban area, the AV TEASY 3 (Testing and Engineering of Automated Driving SYstems) was used (Figure 2). This AV was equipped with sensors by the Institute of Automotive Engineering of Technische Universität Braunschweig. In ad-

1 Message with detailed road topology information used by RLT service: ETSI I2C message type about the road topology, see ETSI (2018). 

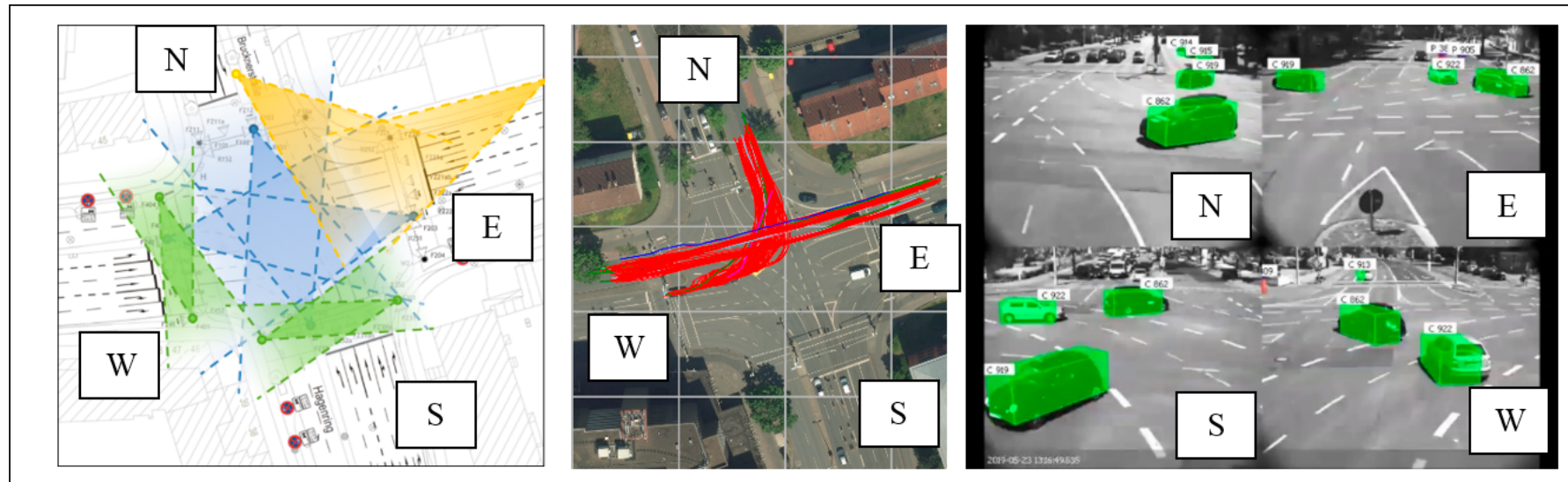

Figure 1. Schematic representation of AIM Research Intersection, its sensors and detection areas (left): blue: mono-cameras, green and amber: stereo-cameras; one-hour trajectory data sample of the relevant trajectories for the different transport modes cars: red, bicyclists: blue (middle); augmented video scene showing AV TEASY 3 with ID C862 in different camera views (right).



Figure 2. Automated vehicle TEASY 3 with relevant environment sensor systems.

dition to the series production sensors, mid-range radar, and mono camera, the vehicle was equipped with a laser scanner perception system. During the measuring period, it consisted of six object detection Ibeo LUX laser scanners with an opening angle of $110^{\circ}$ as well as two rear-facing SICK LMS511 one-layer scanners dedicated for lane marking detection. The latter were mainly used for referencing and data recording purposes to reconstruct a comprehensive model of the road marking environment. TEASY 3 was capable of sending and receiving $\mathrm{V} 2 \mathrm{X}$ messages.

Precise localisation for in-lane positioning within the intersection was performed by a Genesys ADMA speed inertial measurement unit, obtaining a GPS signal with d-GPS correction via mobile network. Information from the digital infrastructure, such as the traffic light status and status prediction were provided through a SPATem, which was received via WAVE (wireless access in vehicular environments) communication (Flormann et al., 2018). On the actuator side, the vehicle was furthermore equipped with CAN interfaces allowing the reception and transmission of relevant messages and, therefore, a manipulation of the acceleration, brake torque as well as the steering angle.

The vehicle's intelligence was embedded in a multi-layer functional architecture running on a dSpace AutoBox, a rapid control prototyping platform connected to the perception and actuator side. The architecture was comprised of an environment model, behaviour planning, and a control layer. The ADF implemented within this environment were domain and use-case focused and include a highway pilot, automated valet parking applications, and multiple intersection use cases.

\subsection{Explorative observation}

The observation took place at the AIM Research Intersection, in Braunschweig, Germany. Of interest were vehicles turning left from West to North (WN vehicles) as well as the oncoming vehicles from East to West (EW vehicles). One dedicated left turn lane was provided at the intersection while oncoming traffic could choose between two lanes for crossing the intersection, the right and the left lane. The WN vehicles queued up either at the first or the second position. WN and EW vehicles interacted regularly since left turning vehicles and the oncoming traffic accessed the intersection in the same traffic light phase. Therefore, this situation can be called 'conditionally tolerable left turn'. Such situations can be critical. Both interacting vehicles share the same conflict area and, if at least one of the interacting partners does not behave adequately or the resulting gap of oncoming vehicles is assessed falsely by the turning vehicle (or driver), a critical situation may evolve to a severe crash.

The recorded video and trajectory data were reduced to the relevant interaction situations between left turning vehicles from West to North (including the drives with TEASY 3) and oncoming vehicles from East to West. For the purpose of the analysis, matched MV situations were extracted from the data set. In order to ensure comparability of $\mathrm{AV}$ and MV situations, the following extraction criteria were applied:

- Same traffic conditions: The time period of the extraction of MV situations was set to ten minutes before and after the AV situation.

- Grouping by queue position: Since the AV was found to queue either at the first or second position, MV also need- 
ed to be positioned first or second in the queue. Further, all $\mathrm{AV}$ and $\mathrm{MV}$ of these relevant cases that queued up at first position came to a standstill before crossing the conflict area.

- Grouping by lane of the oncoming traffic: The oncoming traffic was characterised by vehicles adopting either the right lane to pass the intersection or the left lane. A separation of the target lane of the left turning vehicle was not considered due to its negligible impact on the conflict area.

To compare the situations of AV and MV, the traffic area for left turning vehicles was separated into ten sections including different driving scenarios: (i) approaching the intersection, (ii) entering the conflict area with the oncoming traffic and (iii) leaving the intersection (Figure 3). Each of the sections was chosen to be equidistant. Of all relevant WN situations, the interacting trajectories were selected enriching the video data with information about the queue position (first/second position) and lane positioning (right/left lane) of the oncoming traffic. Relevant variables were time stamp, road user kinematics (position, speed, acceleration), the surrogate safety indicator PET, accepted and non-accepted net time gaps, the distance covered to reach the conflict point $\left(\mathrm{d}_{\mathrm{cp}}\right)$ in the moment of first acceleration or brake and the corresponding time needed to arrive at the conflict point $\left(t_{\mathrm{cp}}\right)$. In order to investigate difference of $\mathrm{AV}$ and $\mathrm{MV}$ approaching and leaving the common conflict point, two different cases were considered: the distance covered to reach the conflict point $d_{c p}( \pm a)$ and the time needed to arrive at the conflict point $t_{\mathrm{cp}}( \pm \mathrm{a})$ given the acceleration of the vehicle. The variable " $\pm \mathrm{a}$ " was the independent parameter acceleration indicating either a deceleration $(-a)$ or an acceleration manoeuvre $(+a)$. Due to acceleration noise of the underlying trajectory data, several thresholds were considered: First, the threshold for decelerating was set to $-1.3 \mathrm{~m} / \mathrm{s}^{2}$. The threshold to count an event as an accelerating manoeuvre was set to $+1.0 \mathrm{~m} / \mathrm{s}^{2}$. The absolute values of both values differed slightly since vehicles usually accelerate more moderately than they decelerate. Second, accelerating and decelerating are not singular events, instead, they are kinetic processes lasting some time to change the speed of the vehicle significantly due to its inertia. Therefore, a speed changing manoeuvre was considered and counted as accelerating/decelerating process only in case the accel- erating/decelerating thresholds were exceeded for at least $0.7 \mathrm{~s}$. All variables were derived from the trajectory data. The kinematic variables were computed for the ten sections on the WN relations (see Figure 3).

\subsection{Analysis}

Methods of descriptive and inferential statistics were applied to the underlying AV and MV data. The following significance tests were applied:

- All variables selected for answering the research questions in section 2.3 were tested for normality of the residuals by applying the Shapiro-Wilk test. Some of the data samples were significant to reject the normality assumption.

- PET, time gap acceptance/non-acceptance, $d_{c p}, t_{c p}$ :

- Due to the different number of sample sizes and the violations of normality, a parametric T-test to analyse the differences between AV and MV behaviour could not be applied. Thus, the non-parametric Mann-Whitney-U test was used.

- Speed and acceleration data:

- Speed and acceleration data were tested for homoscedasticity. In most cases, this requirement was not met.

- Due to the different sample sizes and the violation of the homoscedasticity condition, a parametric ANOVA-test could not be applied. Therefore, the Kruskal-Wallis-H test was applied.

\section{RESULTS}

Generally, all tests were applied with a level of significance of $\alpha<0.05$. In case of the post hoc tests, the Bonferroni correction was applied.

\subsection{Interaction behaviour}

For this analysis, the interaction behaviour was analysed based on the PET, accepted/non-accepted gaps as well as the distance to arrive at the conflict point $\left(\mathrm{d}_{\mathrm{cp}}\right)$ and the time needed to arrive at the conflict point $\left(t_{\mathrm{cp}}\right)$ for the first acceleration/deceleration manoeuvre of the left turning vehicle.

\subsubsection{PET}

In Figure 4 , the derived PET histogram of all situations $(n=1235)$ of left turning vehicles (including the drives with

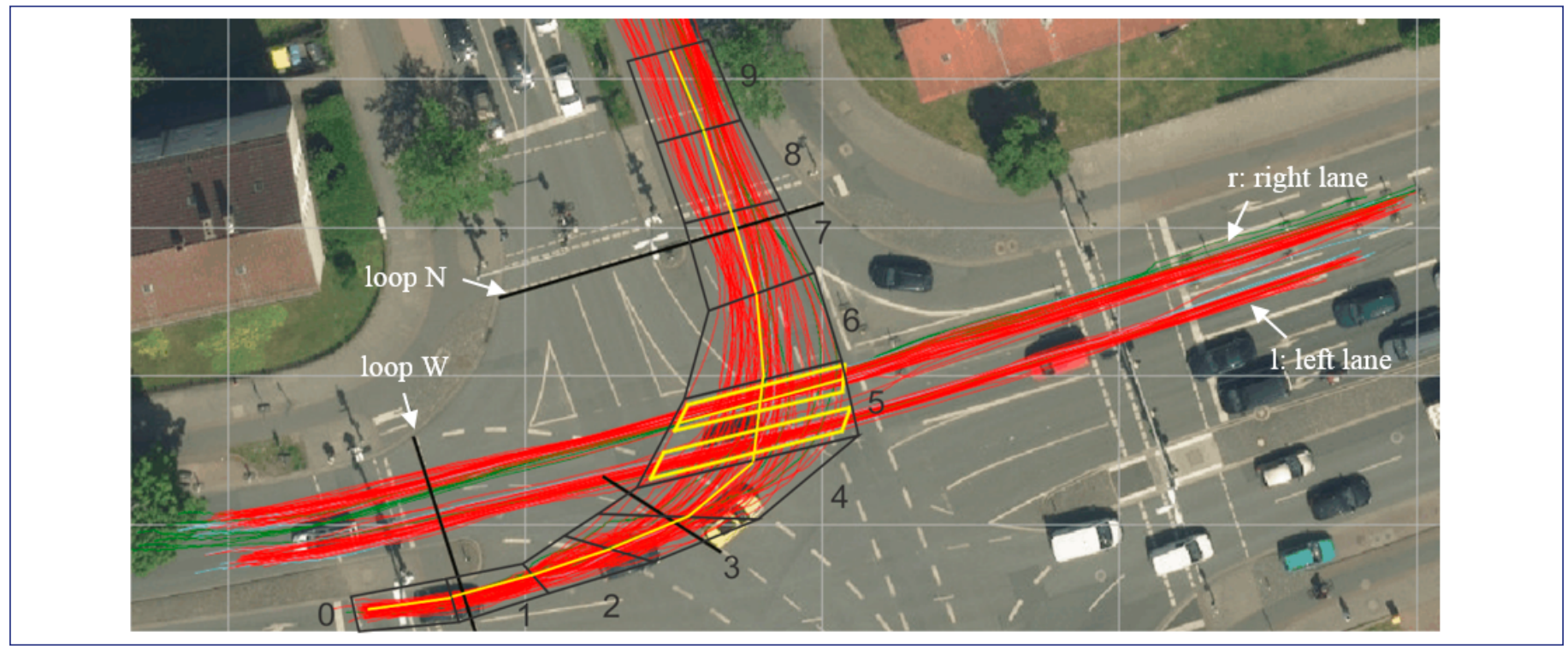

Figure 3. Division of the relevant parts of the intersection in 10 sections for vehicles turning left from West to North (sections 0-4: approaching; section 5: conflict area; sections 6-9: leaving). The red curves are sample trajectories for the WN vehicles and the EW vehicles. The yellow boxes define the conflict area; the piecewise yellow lines define the equidistant sections. The long black lines mark two relevant loops (Western and Northern loop) for distance-time diagram computation. 
the $\mathrm{AV}$ ) interacting with vehicles of the oncoming traffic are shown: $\mathrm{M}=4.25 \mathrm{~s}, \mathrm{SD}=3.03 \mathrm{~s}, \mathrm{Mdn}=3.33 \mathrm{~s}$. For each PET, it was expected that the interaction took place between the vehicle pair that showed the minimum PET. The most frequent PET values lay between 1.5 and 2.0 seconds, whereas the frequency of PET values larger than 2.0 seconds tended to decrease. PET values of less than 1.0 second appeared to be relatively rare.

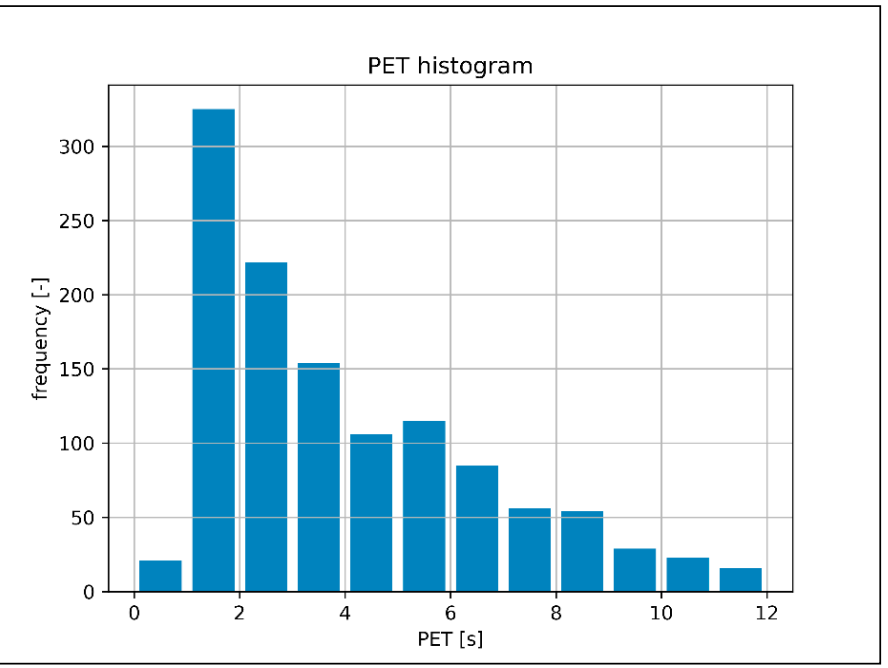

Figure 4. PET histogram of all interactions between left turning vehicles with oncoming traffic during the whole measuring period.

In the left of Figure 5, the PET values for AV and MV (baseline) situations are plotted. The AV showed PET values with a Mdn $=6.35 \mathrm{~s}$, whereas the PET values for MV drives showed a Mdn $=1.73$ s. In this example, a comparison of the PET values applying Mann-Whitney-U test $(\mathrm{U}=20.0, \mathrm{p}<.001, \mathrm{r}=.756)$ revealed a significant difference between AV and MV.

Based on these results, it was aimed to investigate, whether the position in the queue of left turning vehicle and/or the lane use of the vehicles of the oncoming traffic influence the PET distribution. For instance, in the right of Figure 5, the box plots of the PET values regarding the position of the queue of the left turning vehicle are shown. Here, significant differences $(\alpha=0.0125)$ were found for the first and second positions of $\mathrm{MV}(\mathrm{U}=86.0, \mathrm{p}<.001, \mathrm{r}=.514)$ with $\mathrm{PET}$
Mdn $=1.64 \mathrm{~s}$ (first position) and $\mathrm{Mdn}=2.94 \mathrm{~s}$ (second position), and between the first positions of the AV compared to the first position of MV $(\mathrm{U}=11.0, \mathrm{p}<.001, \mathrm{r}=.681)$ with PET $M d n=6.23 \mathrm{~s}$ vs. $\mathrm{Mdn}=1.64 \mathrm{~s}$ and between the second position of the AV and the second position of $\mathrm{MV}(\mathrm{U}=1.0, \mathrm{p}<.001$, $r=.838$ ) with PET Mdn $=7.36 \mathrm{~s}$ vs. $\mathrm{Mdn}=2.94 \mathrm{~s}$ ). The differences between the first and the second position of the AV were not significant $(\mathrm{U}=47.0, \mathrm{p}=.019, \mathrm{r}=.404)$. The PET values of $\mathrm{AV}$ and MV were approximately 1.1-1.3 seconds larger when the vehicles queued up at the second position compared to the first position. Lane use of the oncoming traffic did not affect the PET distribution within the groups.

\subsubsection{Accepted/non-accepted time gaps}

In Figure 6, the accepted and non-accepted time gaps of the left turning vehicle to intersect the oncoming traffic flow are plotted $(\alpha=0.0125)$. On the left, the results considering the whole data set are shown. No significant difference was found between non-accepted time gaps of AV and MV by applying Mann-Whitney- $U$ test $(\mathrm{U}=14610.0, \mathrm{p}=.274)$. Moreover, in case of the AV, no accepted time gap was found in the data, but the time gaps accepted by MV (Mdn = 1.54s) significantly differed from their non-accepted time gaps $(\mathrm{Mdn}=6.27 \mathrm{~s}$ ) $(\mathrm{U}=252.0, \mathrm{p}<.001, \mathrm{r}=.575)$. On the right, the results for non-accepted time gaps are shown for vehicles that had come to a full-stop before turning left. Therefore, only the vehicles that queued at the first position were considered and no significant difference were revealed.

\subsubsection{Distance to conflict point $\left(\mathrm{d}_{\mathrm{cp}}\right)$ and time to conflict point $\left(t_{c p}\right)$}

\section{$\underline{\mathrm{d}}_{\mathrm{cp}}(-\mathrm{a})$ and $\mathrm{t}_{\mathrm{cp}}(-\mathrm{a})$ :}

In Figure 7 , the box plots of the $\mathrm{d}_{\mathrm{cp}}(-\mathrm{a})$ (left) and the corresponding $\mathrm{t}_{\mathrm{cp}}(-\mathrm{a})$ (right) values for the first braking manoeuvre of the AV and MV are shown $(\alpha=0.05)$. The values for $d_{c p}(-a)$ did not reveal any significant difference between AV and MV $(\mathrm{U}=392.0, \mathrm{p}=.112)$. The values for $\mathrm{t}_{\mathrm{cp}}(-\mathrm{a})$ of the AV differed significantly from MV $(\mathrm{U}=160.5, \mathrm{p}<.001, \mathrm{r}=.554)$ in the moment of first braking. Also, the values for $d_{c p}(-a)$ and $t_{c p}(-a)$ for MV had a larger variance than the ones for the AV.

$\underline{\mathrm{d}}_{\mathrm{cp}}(+\mathrm{a})$ and $\mathrm{t}_{\mathrm{cp}}(+\mathrm{a})$ :

In Figure 8 , the box plots of the values for $d_{c p}(+a)$ (left) and $\mathrm{t}_{\mathrm{cp}}(+\mathrm{a})$ (right) in the moment of first acceleration of the AV and MV are shown $(\alpha=0.05)$. The values for $d_{c p}(+a)$ between

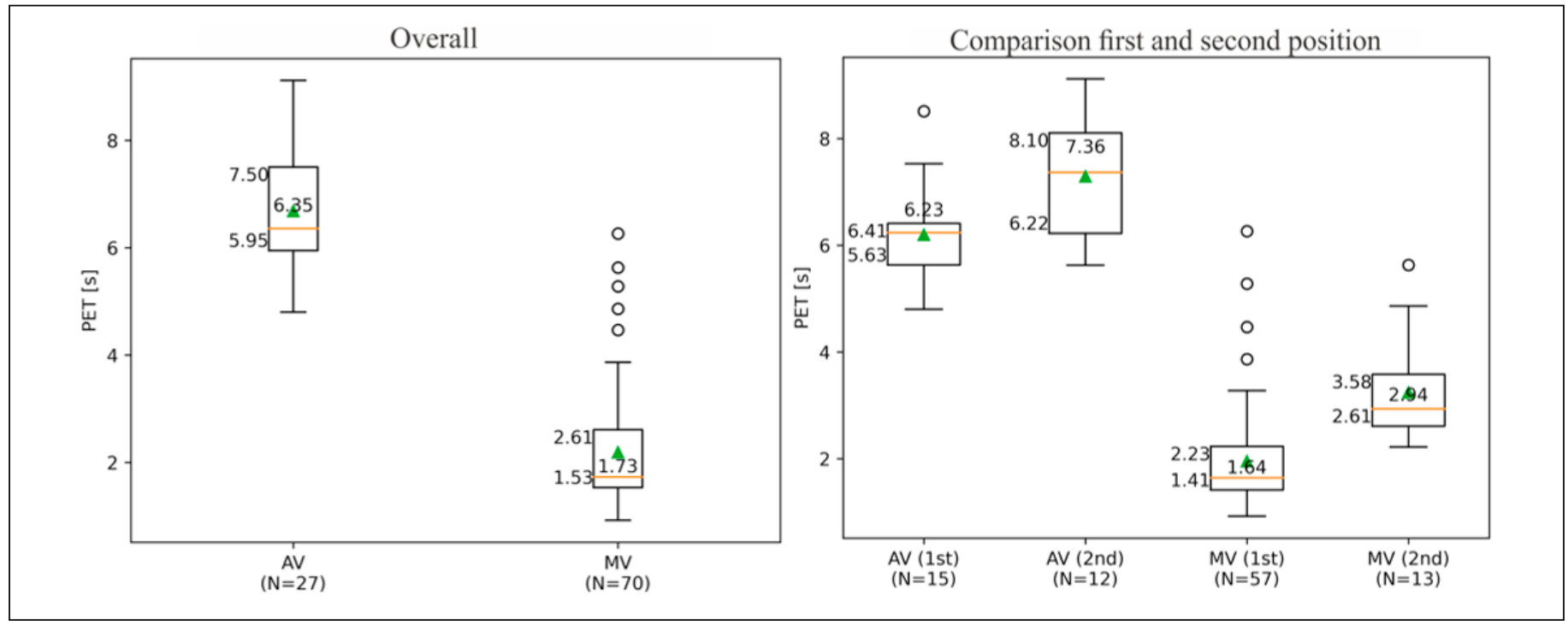

Figure 5. Box plots of the PET values of the 70 baseline MV (Mdn = 1.73s) and $27 \mathrm{AV}(\mathrm{Mdn}=6.35 \mathrm{~s})$ situations (left); box plots of the PET values with regard to the position of the left turning vehicle in the queue; the orange line shows the medians and the green triangles the positions of the means. 
$\mathrm{AV}$ and MV did not differ significantly $\left(\mathrm{Mdn}_{\mathrm{AV}}=9.76 \mathrm{~m}\right.$ vs. $\left.\mathrm{Mdn}_{\mathrm{MV}}=8.42 \mathrm{~m} ; \mathrm{U}=638.0, \mathrm{p}=.063, \mathrm{r}=.164\right)$. Furthermore, the values for $t_{c p}(+a)$ were significantly different between $\mathrm{AV}$ and $\mathrm{MV}$ with $\mathrm{Mdn}=3.86 \mathrm{~s}$ for $\mathrm{AV}$ vs. $\mathrm{Mdn}=2.78 \mathrm{~s}$ for $\mathrm{MV}$ $(\mathrm{U}=393.5, \mathrm{p}<.001, \mathrm{r}=.402)$.

\subsection{Kinematic behaviour}

In Figure 9, the speed of all $27 \mathrm{AV}$ cases as well as 27 (out of 70) randomly chosen MV cases are plotted over the distance travelled within the area covered by the loops $\mathrm{W}$ and $\mathrm{N}$ at the intersection. In case of the AV, speed decreased when approaching the inter-

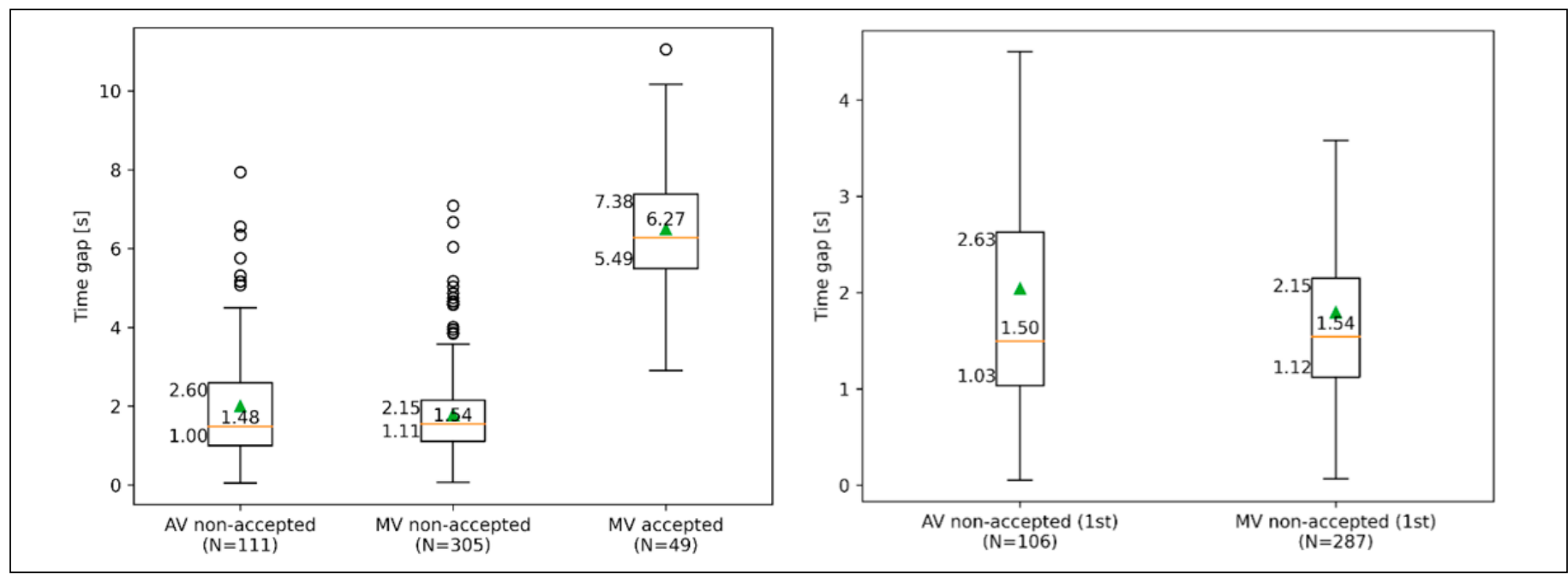

Figure 6. Accepted/non-accepted time gaps for AV and MV: the complete relevant data set with $27 \mathrm{AV}$ and $70 \mathrm{MV}$ cases (left); only the cases with the $\mathrm{AV}$ and $\mathrm{MV}$ at first position of the queue (right); the orange lines represent the medians and the green triangles the means.

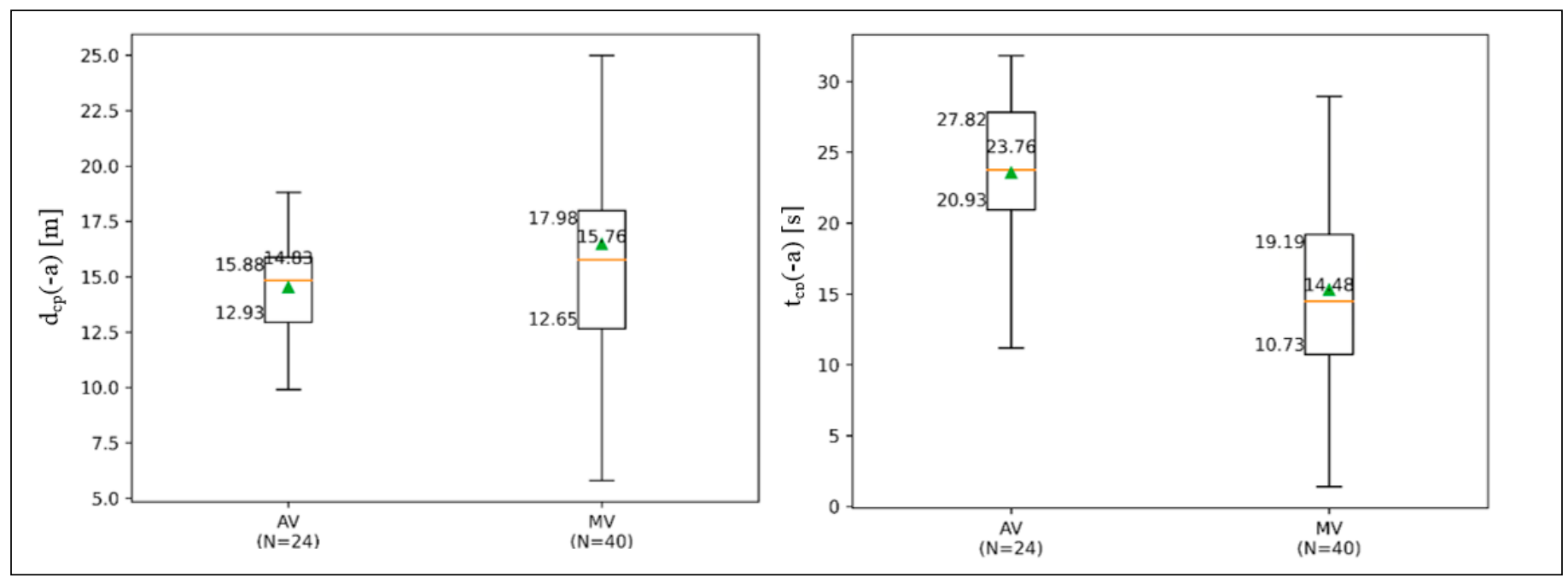

Figure 7. Distance to conflict point and time to conflict point in the moment of the first braking over the whole baseline MV and AV data set: $d_{c p}(-a)(l e f t) ; t_{c p}(-a)$ (right); the orange lines represent the medians and the green triangles the means.

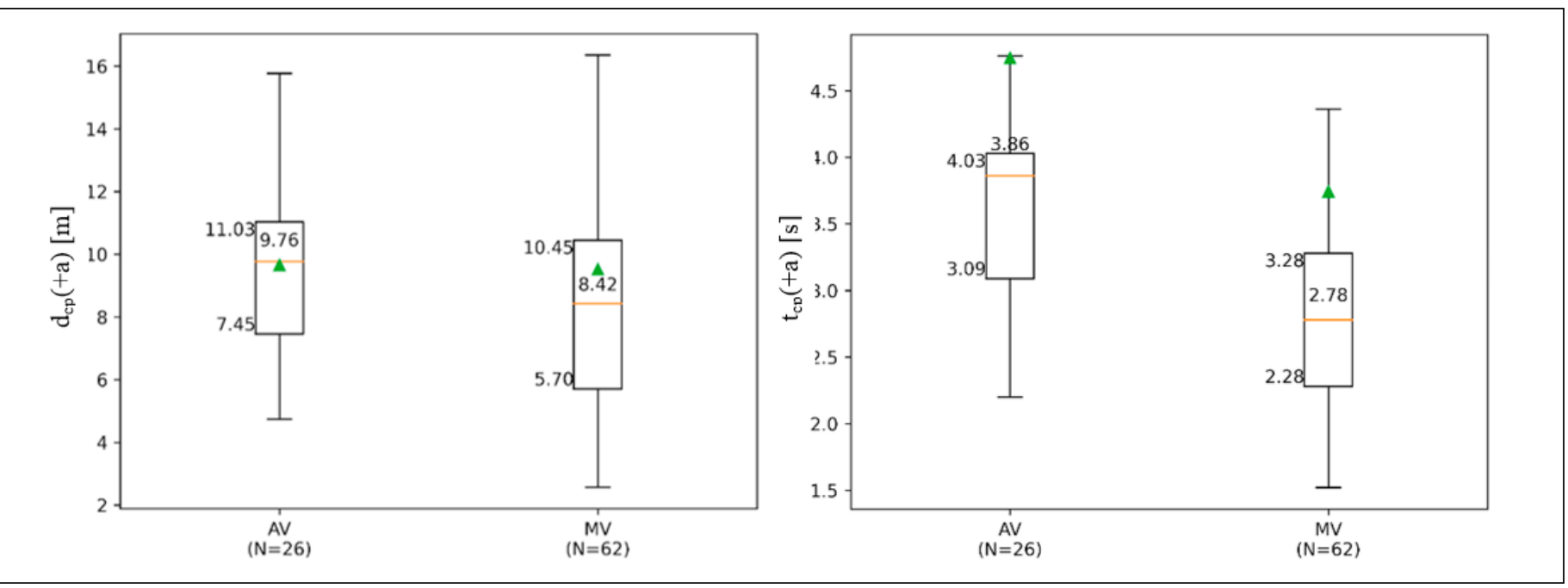

Figure 8. Distance to conflict point and time to conflict point in the moment of the first acceleration over the whole baseline MV and $A V$ data set: $d_{c p}(+a)(l e f t) ; t_{c p}(+a)$ (right); the orange lines represent the medians and the green triangles the means. 

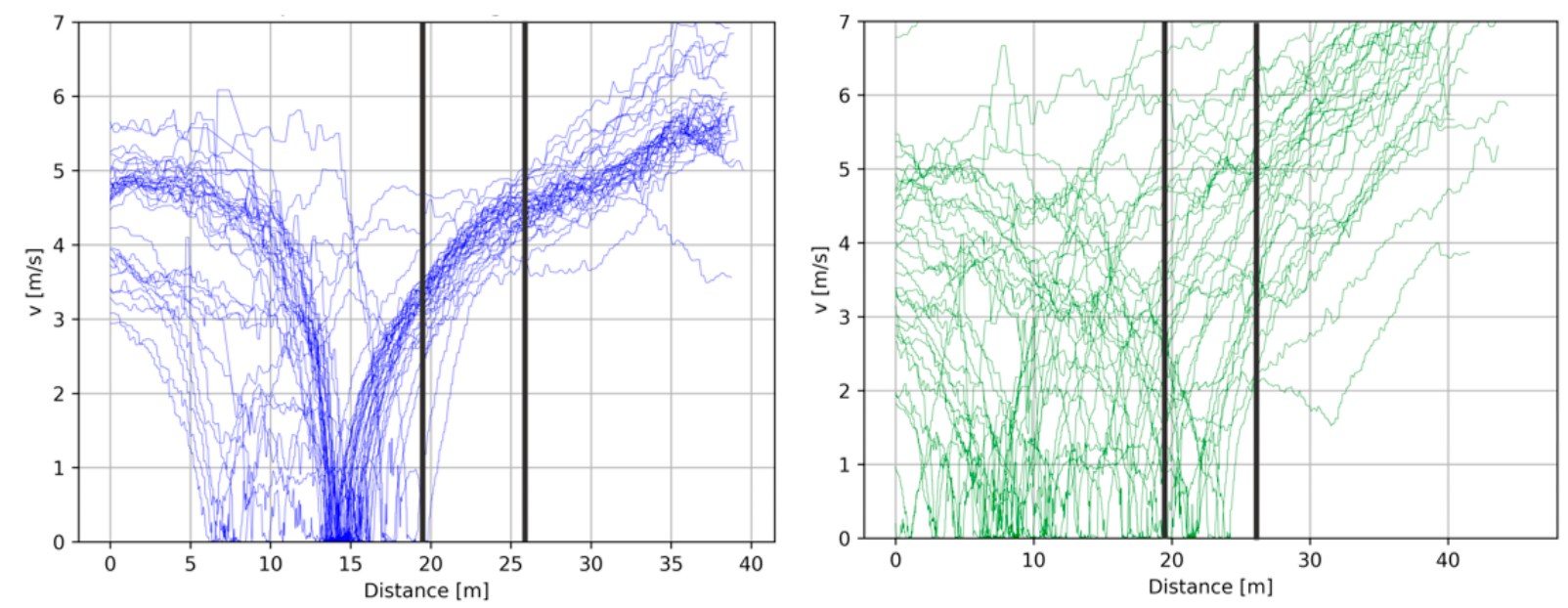

Figure 9. Distance-speed diagrams of the AV drives (left) and MV drives (right) between the loops W and N. The vertical black lines represent the conflict area.
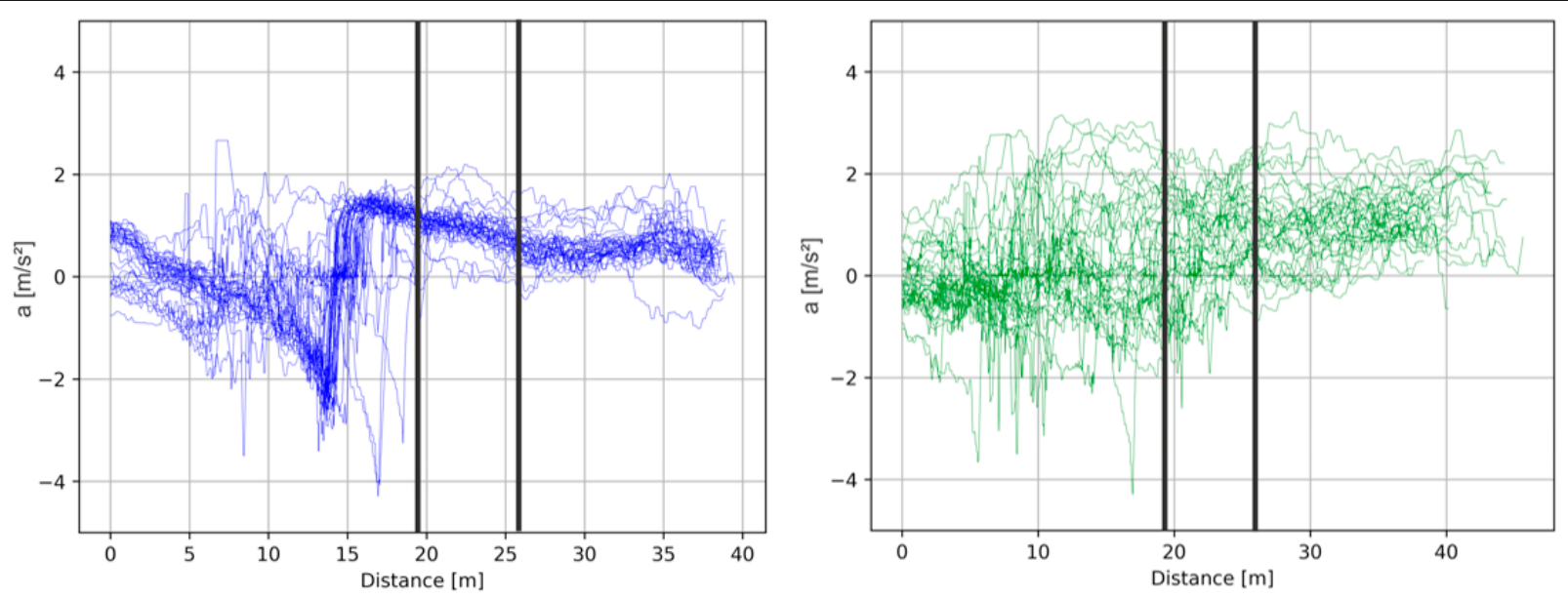

Figure 10. Distance-acceleration diagrams of AV drives (left) and MV drives (right) vehicles between the loops W and N. The vertical black lines represent the conflict area.
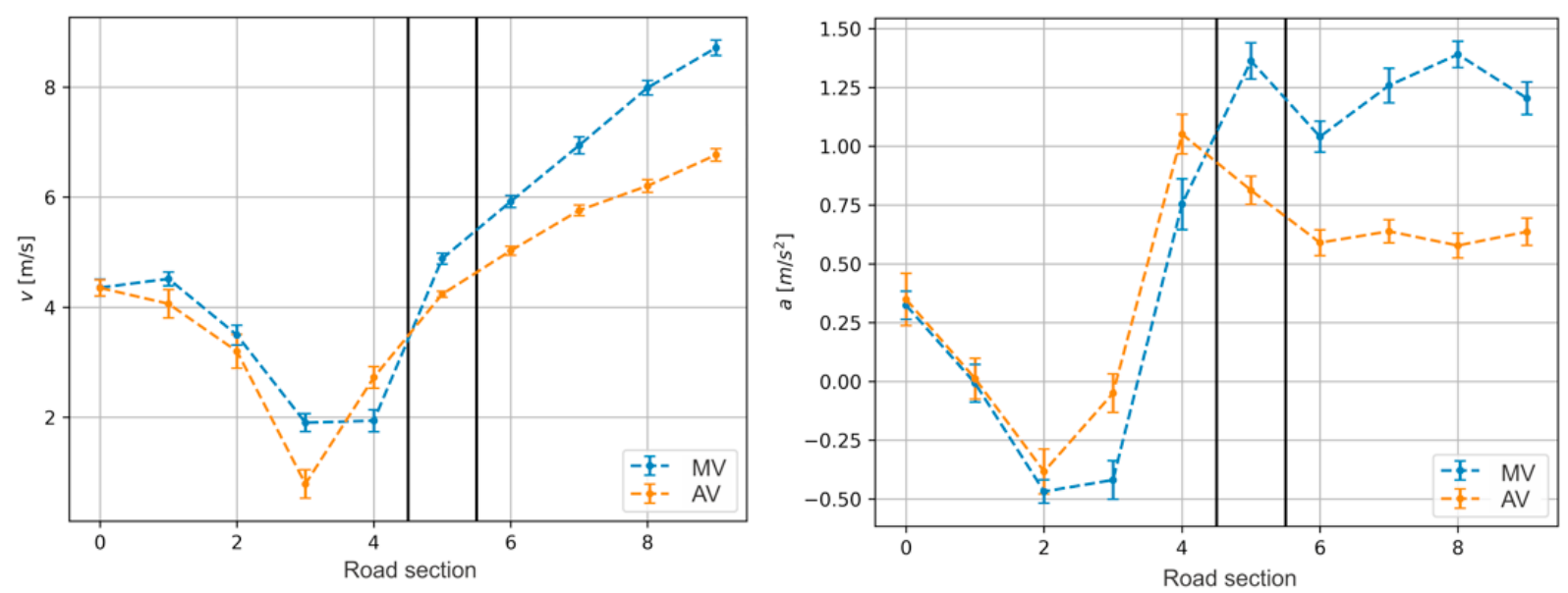

Figure 11. Kinematic mean values of the AV and the baseline MV drives and their standard errors in the defined sections 0-9: mean speeds and their standard errors (left); mean accelerations and their standard errors (right); the vertical black lines mark the conflict area in section 5 .

section and increased when exiting. Speed around $0 \mathrm{~m} / \mathrm{s}$ marked a full-stop and/or queueing up (i.e. lower distances from the start of the sequence) before accelerating to leave the intersection. In case of MV, speed decreased when approaching the intersection, but the distance to the conflict point when acceleration to leave the intersection had a larger variance than for the AV.
In Figure 10, the accelerations of the $27 \mathrm{AV}$ and $27 \mathrm{MV}$ cases are plotted over the distance travelled. In case of the $\mathrm{AV}$, the acceleration followed a certain pattern: decelerating when approaching the intersection and accelerating again when leaving the intersection. In the MV case, no obvious pattern was identified. 
The kinematic behaviour of the AV and MV was analysed in more detail and characterised by their mean speed and acceleration in the ten road sections (see Figure 3 ). The ten road sections were categorised into approaching (0-4), conflict area (5), and leaving (6-9). In Figure 11, the kinematic values (mean speed and acceleration) and standard errors of the 27 $\mathrm{AV}$ and $70 \mathrm{MV}$ drives are shown.

In Figure 11 (left), the mean speed of the AV and MV are shown. The mean speed of MV was higher than the mean speed of the AV in the approaching sections (0-3), the conflict area in section 5, and the leaving sections (6-9). In the queueing area before the conflict point, section 4, the mean speed of MV was lower than the mean speed of the AV. The application of the Kruskal-Wallis-H test $(\alpha=0.005)$ revealed no significant main effect between the speed of AV and MV $(\mathrm{H}=0.691, \mathrm{DF}=9, \mathrm{p}=.406)$.

In Figure 11 (right), mean acceleration of the AV and MV are shown. Mean acceleration of MV and the AV in the approaching sections (0-2) were similar, but in the subsequent sections 3 and 4, accelerations of the $\mathrm{AV}$ were higher than acceleration of MV. Beginning with the conflict zone in area 5, the effect reversed: acceleration of $\mathrm{MV}$ were higher than acceleration of the AV. The application of the Kruskal-Wallis-H test $(\alpha=0.005)$ revealed no significant main effect between the acceleration of $\mathrm{AV}$ and $\mathrm{MV}(\mathrm{H}=0.823, \mathrm{DF}=9, \mathrm{p}=.364)$.

\section{INTERPRETATION AND DISCUSSION}

The objective of the study was to investigate the differences between AV and MV turning left with oncoming traffic based on real interaction data. At the AIM Research Intersection, video-based trajectory data was recorded and the performance of the AV was compared to MV executing the same manoeuvre. The derived trajectories of the AV and MV were analysed regarding the influence of situational and kinematic factors on traffic safety. For instance, it appeared that the AV showed a conservative left turning behaviour leading to safe PET distributions. Although the kinematic behaviour of AV and MV did not differ significantly, some variables (e.g. time gap, time/distance to arrive at the common conflict point) showed significant differences under some circumstances. Also, the queuing position of the left turning vehicle as well as the lane adopted by the oncoming traffic may affect turning behaviour, and hence traffic safety.

\subsection{Interaction behaviour}

The results showed that the ADF of the AV had a conservative behaviour conducting the conditionally tolerable left turns at this specific urban intersection: PET values of the AV and MV groups were on opposite ends of the PET distribution. PET values of the AV were higher than PET values of MV indicating a safe left turn manoeuvre of the AV. The AV waited for a longer period of time before entering the conflict area and leaving the intersection. Also, it was found that PET values of MV and $\mathrm{AV}$ were more than a second apart when queuing up at the second position than in case of the first position. A reason for this may be the missing line of sight of the oncoming traffic by the left turning vehicle placed at the second position. Technically, the AV was capable of intersecting oncoming traffic, this case did not occur when recording the data due to the high safety demands and the limited sensor range. The largest non-accepted gap of the $\mathrm{AV}$ to intersect the oncoming traffic was about 8 seconds. In contrast to our study, in which the AV always came to a full-stop before turning-left, in Shu et al. (2020)-which was a simulation-based study-the AV approached further, closer to the conflict point at very low speeds and took a shorter path to leave the intersection in case the gap to vehicles of oncoming traffic was large enough.
It appears that the values for the time to arrive at the conflict point at the moment of first braking were approximately 9.7 seconds larger for the AV than MV, while the distance to reach the conflict point was non-significantly a meter shorter indicating later braking of the AV. The AV approached the intersection at lower speeds than MV. This may be due to the ability to better anticipate the behaviour of the vehicle in front or lacking relevant information. The variance of the values of the distance to the conflict point was larger for MV compared to AV indicating that (i) MV braked despite the conflict point and thus, the moment of first braking appeared to be arbitrary, and (ii) human drivers adapt different driving behaviours in different conditions (e.g. traffic light phases, anticipated gap). MV approached the intersection faster than the AV. This may indicate that in a first queue position human drivers brake more often. It may be seen as a strategy to not lose the momentum just in case stopping is not necessary. This behaviour may allow utilizing smaller gaps. The AV braked just once and usually to a full stop. Also, the ADF of the AV in question did not distinguish between the occupation of the lane of the oncoming traffic. In case vehicles were present, all lanes were set to be occupied. This also indicates that the AV appears to stop in the same area independently of the adopted lane of the oncoming traffic while MV drivers adapt to the oncoming traffic allowing shorter gaps to intersect the traffic flow. In consequence, the AV appears to reject smaller gaps and to wait longer before entering the conflict area. In turn, this may lead to a lower efficiency. In Shu et al. (2020), it is stated that the waiting time before getting in the conflict area is longer when oncoming vehicles drive at a lower speed and shorter when oncoming vehicles are faster (which can be interpreted as a larger time to arrive at the conflict point). The typical qualitative behaviour regarding the speed leaving the intersection were comparable to our results and showed human-like behaviour. Although the ADF of TEASY 3 considered the changes in speed and acceleration of the oncoming traffic, it did not distinguish between the adopted lanes of the oncoming traffic. Its ADF used map related stopping positions and it showed potential for dynamic optimisation of the path to cross the conflict area. This is the main cause that TEASY 3 was less efficient than MV.

In case of the distance to the conflict point in the moment of the first acceleration, no significant differences between AV and MV were found. The time of the AV to reach the conflict point was a second longer than MV. This trend may indicate that the AV accelerated earlier than MV, but with lower acceleration and lower speed. Human drivers are better in recognizing road users in the intersection than the limited sensor range of the AV allows for. Therefore, it is expected that human drivers anticipate the left turn situation better than the AV. Also, the acceleration function of the AV in question was limited to allow the safety driver to react adequately in case a faulty acceleration occurred. In general, the AV approached and left the conflict point at a lower speed than MV. The means of the time to arrive at the conflict point at first acceleration are larger than the medians. This indicates a strong asymmetry of the underlying distributions.

In general, it can be stated that the driving behaviour of the $\mathrm{AV}$ did not differ completely from MV (i.e. the AV behaviour can be seen as a subset of the driving behaviour of human drivers). The human anticipation performance appeared to be-particularly in terms of the specific interaction with oncoming traffic-better than the ADF in question. The differences are not surprising as liability is an issue as well as the fact that the vehicle in question represented a prototypical state of implementation in an experimental vehicle. However, it appears that the ADF of the examined AV showed potential to increase its driving efficiency in terms of lowering the implemented subroutines with regard to safety-related 
parameters such as PET and gap acceptance. The currently implemented safety margin may be decreased significantly while maintaining a comparable safety level, for instance, by applying and safeguarding powerful AI methods (e.g. deep learning as proposed by Liu et al., 2020) and stochastic Markov decision process methods (e.g. Shu et al., 2020) in the decision-making process of the ADF.

\subsection{Kinematic behaviour}

It can be stated that MV-with different car and driver typesshow a more or less arbitrary behaviour while the AV behaviour appears to be predictable. On the other hand, it appears, the AV always behaved in the same way, while MV showed a variety of behaviour depending on the current traffic situation. For instance, MV intersected the oncoming traffic up to approximately 8 meters before the conflict area when gaps were suitable, whereas the AV always stopped. Also, unlike $\mathrm{MV}$, the acceleration of the AV was limited in order to give the safety driver a chance to react adequately in case of a faulty behaviour of the vehicle.

When looking at the different phases of conducting a left turn, the AV decreased its speed when approaching the intersection, maintained a low speed until coming to a fullstop and accelerated again to leave the intersection. These real-data findings are qualitatively similar to the results of a simulation-based study presented by Shu et al. (2020). They found that in all left turn scenarios, the AV approached the intersection at a relatively high speed, decelerated, and maintained a low speed creeping forward until the intention of the oncoming vehicles became certain (either stopping or accelerating in dependence on the gap size of the vehicles of the oncoming traffic).

\subsection{Research question and hypotheses}

The acceleration and speed of the AV in comparison to human drivers did not differ compared to MV. The ADF of this specific AV in question did not behave completely different from human drivers. However, the AV showed a tendency to approach the intersection and the conflict point slower than human drivers. On the other hand, the AV accelerated earlier, but more steadily than MV to leave the conflict area and the intersection. The hypotheses $\mathrm{H} 1$ and $\mathrm{H} 2$ meaning the AV approached, passed, and left the intersection slower and with less acceleration than MV were rejected. However, the AV showed a tendency to fulfil these expectations. Also, in most cases-as the distance-speed and distance-acceleration figures showed-the acceleration and speed behaviour of the AV occurred widely predictable. This, on the one hand, gives road users the chance to anticipate the outcomes of future traffic situations better than in case of human drivers. On the other hand, a higher rate of deployed AV in traffic will allow for more precise coordinated and cooperative driving, resulting in increased traffic efficiency. In case of several different AV with different ADFs, the results are expected to be different. Concerning both, the moment of braking before the conflict point was realised in almost all cases later compared to MV. The AV accelerated at an earlier point than MV to leave the conflict and intersection area. An explanation may be that the AV drove more slowly and with less acceleration in order to minimise uncertainties of the sensor-based road user detection and prediction. Hypothesis $\mathrm{H} 3$ was confirmed with regard to the time needed to arrive at the conflict point. The AV needed significant more time than MV at the moment of first braking/acceleration due to its lower speed and acceleration. In general, the AV showed a tendency to brake later (i.e. at a larger distance to the conflict point) than MV. On the other hand, the AV showed a tendency to accelerate earlier (i.e. at a greater distance before the conflict point) than MV. Hypothesis $\mathrm{H} 3$ with regard to the distance to the conflict point was rejected. Hypothesis $\mathrm{H} 4$ was confirmed, since the AV showed a less critical interaction behaviour than MV regarding the PET. But it was not expected that the differences were so prominent between the AV and MV interacting with oncoming traffic. Lastly, hypothesis $\mathrm{H} 5$ could not be addressed, since no cases were recorded in which the AV accepted single gaps in oncoming traffic.

\section{CONCLUSIONS AND FUTURE PROSPECTS}

Conditionally left turning in oncoming traffic at an urban signalised intersection is a challenging task for human drivers and ADF of AV. The results of this paper show that the $\mathrm{AV}$ in question was capable of turning left without violating traffic regulation rules. Its driving behaviour did not show significant differences to human drivers, except for the gap acceptance. Additionally, the driving behaviour of the AV may be characterised as predictable. This may change when the penetration rate increases and several AV of different OEMs enter the market. At the same time, a higher deployment rate may also result in an increase of traffic efficiency. Significant differences were identified in terms of safe driving: Unlike $\mathrm{MV}$, the AV turned left in a conservative and safe way. Also, the AV did not enter the conflict area in dependence of the lane adopted by the vehicles of the oncoming traffic, which led to the low traffic efficiency in comparison to MV. Eventually, these results may help to improve and mature the ADF in order to increase traffic efficiency while maintaining a high level of safety. The identified safety margin could be significantly decreased. However, the results presented here should be handled carefully, since the amount of cases considered was very low. Also, more situational factors need to be considered, which requires more data, particularly with AV to come to more sound conclusions. Our future work will deal with improving the current set of ADF by including the results of this investigation, and testing them maintaining safety, but increasing traffic efficiency.

\section{Acknowledgements}

We would like to gratefully acknowledge the Germany Federal Ministry of Transport and Digital Infrastructure (BMVI) to grant the project "Digitaler Knoten 4.0" and the project "ViVre", without this research would have not been possible.

\section{REFERENCES}

@City. (2020, November 11). Retrieved from https://www.atcityonline.de/project/ Projekt.html.

Bärgmann, J., Smith, K., Werneke, J. (2015). Quantifying drivers' comfort-zone and dread-zone boundaries in left turn across path/opposite direction (LTAP/OD) scenarios. Transportation Research Part F 35 (2015), 170-184. Retrieved from https://www.sciencedirect.com/science/article/pii/ S1369847815001540.

Berman, B. (2020, January 10). Interview: VW's hip, all-electric ID Buzz van is a platform for its self-driving efforts. Retrieved from https://electrek.co/2020/01/10/interview-vws-hip-all-electricid-buzz-van-is-a-platform-for-its-self-driving-efforts/

Brunner P., Denk, F., Huber, W. and Kates, R. (2019). Virtual safety performance assessment for automated driving in com-plex urban traffic scenarios, In Proceedings of the 2019 IEEE Intelligent Transportation Systems Conference (ITSC), New Zealand.

Chan, C.-Y. (2006). Characterization of Driving Behaviors Based on Field Observation of Intersection Left turn Across-Path Scenarios. In IEEE Transactions on Intelligent Transportation Systems, Vol. 7, No. 3, September 2006.

Dotzauer, M., Preuk, K., Schießl, C., Gesslbauer, T., Patz, D. (2017). Kooperatives Forschungsvorhaben: Autonome und menschliche (Beinahe-)Unfälle. Final report. 
ETSI. (2018). ETSI TS 101 539-2 V1.1.1 (2018-06) Intelligent Transport Systems (ITS); V2X Applications; Part 2: Intersection Collision Risk Warning (ICRW) application requirements specification, 2018.

EURO NCAP. (2019). European New Car Assessment Programme (Euro NCAP) - Test protocol - AEB Car-to-Car systems, Version 3.0.2. Retrieved from https://cdn.euroncap.com/media/56143/ euro-ncap-aeb-c2c-test-protocol-v302.pdf

EURO NCAP. (2017). Euro NCAP2025 Roadmap - In pusuit of Vision Zero. Retrieved from https://cdn.euroncap.com/media/30700/ euroncap-roadmap-2025-v4.pdf.

EU Regulation. (2019, November 27). Regulation (EU) 2019/2144 of the European Parliament and of the council. Retrieved from https://eur-lex.europa.eu/legal-content/EN/TXT/PDF/?uri=CE LEX:32019R2144\&from=EN.

Fairfield, N., Herbach, J. S., Furman, V. (2014). Remote assistance for autonomous vehicles in predetermined situations. US patent US9720410B2. Retrieved from https://patents.google.com/ patent/US9720410B2/en.

Fleming C. (2016, July 26). Tesla car mangled in fatal crash was on Autopilot and speeding, NTSB says. 2016. Retrieved from: http://www.latimes.com/business/autos/la-fi-hy-autopilotphoto-20160726-snap-story.html\#.

Flormann, M., Sonka, A., Henze, R. (2018). Automated and Connected Driving in Urban Scenarios. In Proceedings of ADAPTIVE 2018: The Tenth International Conference on Adaptive and Self-Adaptive Systems and Applications, 18.-22.02.2018, 66-68.

Gimm, K., Knake-Langhorst, S., Dotzauer, M., Urban, U., Arndt, R. (2016). Increasing cyclist safety with infrastructural supported cooperative ADAS in EU XCYCLE by extending test site AIM Research Intersection. In Proceedings of the International Cycling Safety Conference 2016, 03.-04. Nov. 2016, Bologna, Italy.

González, D., Perez, J., Milanes, V., Nashashibi, F. (2016). A Review of Motion Planning Techniques for Automated Vehicles. In IEEE Transactions on Intelligent Transportation Systems 17 (2016), No. 4, 1135-1145.

Green J. (2018, March 30). Tesla: Autopilot was on during deadly Mountain View crash. 2018. Retrieved from: https://www. mercurynews.com/2018/03/30/tesla-autopilot-was-on-duringdeadly-mountain-view-crash/.

Graber, W., Sussman, A. (2019, May 14). \$30,000 from a Tesla Robotaxi?...not as crazy as it sounds. Retrieved from https:// medium.com/swlh/30-000-from-a-tesla-robotaxi-not-as-crazyas-it-sounds-dafba4c6923c\#.

Hagenzieker, M. P., van der Kint, S., Vissers, L., van Schagen, I. N. L. G., de Bruin, J., van Gent, P., Commandeur, J. J. F. (2020). Interactions between cyclists and automated vehicles: Results of a photo experiment, Journal of Transportation Safety E Security, 12:1, 94-115, DOI: 10.1080/19439962.2019.1591556.

Hubmann, C., Schulz, J., Becker, M., Althoff, D., Stiller, C. (2018). Automated Driving in Uncertain Environments: Planning With Interaction and Uncertain Maneuver Prediction, IEEE Transactions on Intelligent Vehicles, vol. 3, no. 1, pp. 517, 2018.

Hydén, C. (1987). The development of a method for traffic safety evaluation: the Swedish traffic conflict technique. Doctoral thesis. Lund University, Sweden.

Ismail, K., Sayed, T., Saunier, N. (2010). Automated safety analysis using video sensor: technology and case studies. In Canadian Multidisciplinary Road Safety Conference, Ontario, Canada.

Jenssen, G. D., Moen, T., Johnsen, S. O. (2019). Accidents with Automated Vehicles - Do self-driving cars need a better sense of self? In Proceedings of the 26th ITS World Congress, Singapore, 2019.

Krauns, F., Henze, R., Küçükay, F., Raksincharoensak, P. (2019). Objectification of Automated Driving at Intersections. In Proceedings of the 5th International Symposium on Future Active Safety Technology toward Zero Accidents (FAST-zero '19), September 9-11, 2019.

L3Pilot (2020, November 4). Retrieved from https://www.13pilot.eu/.
Liebner, M., Klanner, F., Baumann, M., Ruhhammer, C., Stiller, C. (2013). Velocity-based Driver Intent Inference at Urban Intersections in the Presence of Preceding Vehicles. In IEEE Intelligent Transportation Systems Magazine 5 (2013), No. 2, 10-21.

Liesner, L. (2017). Automatisierte Funktionsoptimierung von Adaptive Cruise Control. Doctoral thesis, Technische Universität Braunschweig. Shaker Verlag, Aachen.

Liu, T., Mu, X., Huang, B., Tang, X., Zhao, F., Wang, X., Cao, D. (2020). Decision-making at Unsignalized Intersection for Autonomous Vehicles: Left-turn Maneuver with Deep Reinforcement Learning. Retrieved from: https://arxiv.org/ftp/ arxiv/papers/2008/2008.06595.pdf.

Matthaei, R., Maurer, M. (2015). Autonomous driving - a topdown-approach, at - Automatisierungstechnik, 63(3), 155-167.

National Highway Traffic Safety Administration. (2017). Fatal Motor Vehicle Crashes: 2016 Overview. Retrieve from: https://crashstats.nhtsa.dot.gov/Api/Public/ Publication/812456, October 2017.

Noh, S. (2019). Decision-Making Framework for Autonomous Driving at Road Intersections: Safeguarding Against Collision, Overly Conservative Behavior, and Violation Vehicles. IEEE Transactions on Industrial Electronics, Vol. 66, No. 4, April 2019, pp. 3275-3286.

Ohnsman, A. (2020, October 8). Waymo Restarts Robotaxi Service Without Human Safety Drivers. Retrieved from https://www.forbes.com/sites/alanohnsman/2020/10/08/ waymo-restarts-robotaxi-service-without-human-safetydrivers $/$ ? $\mathrm{sh}=35 \mathrm{e} 0 \mathrm{a} 8569 \mathrm{~d} 86$.

Pink O., Becker J., Kammel S. (2015). Automated driving on public roads: Experiences in real traffic. Journal it-Information Technology 2015, 57(4), 223-230. Retrieved from https://www. degruyter.com/view/journals/itit/57/4/article-p223.xml.

Ragland, D., Arroyo, S., Shladover, S. E., Minsener, J. A., Chan, C.-Y. (2006). Gap acceptance for vehicles turning left across on-coming traffic: Implications for Intersection Decision Support design. In Transportation Research Records (TRR) of Transportation Research Board (TRB), 2006.

Rad, S. R., de Almeida Correia, G. H., Hagenzieker, M. P. (2020). Pedestrians' road crossing behaviour in front of automated vehicles: Results from a pedestrian simulation experiment using agent-based modelling. Transportation Research Part F: Traffic Psychology and Behaviour, Volume 69, February 2020, 101-119. doi: 10.1016/j.trf.2020.01.014.

Seo, H.-S., Kim, H.-U., Noh, D.-G., Lee, S.-S. (2013). Algorithm Design and Implementation for Safe Left Turn at an Intersection Based on Vehicle-to-Vehicle Communications. The Journal of Korean Institute of Communications and Information Sciences 38C, Issue 2, 165-171. Retrieved from http://koreascience. or.kr/article/JAKO201310635654533.page

Shu, K., Yu, H., Chen, X., Chen, L., Wang, Q., Li, L., Cao, D. (2020). Autonomous Driving at Intersections: A Critical-Turning-Point Approach for Left Turns. Retrieved from: https://arxiv.org/ pdf/2003.02409.pdf

Sonka, A. M. (2020). Klassifikation und Prädiktion der Verkehrsumgebung für das automatisierte Fahren. Doctoral thesis, Technische Universität Braunschweig. Shaker Verlag, Düren, Germany.

Tarko, A. (2019). Measuring Road Safety with Surrogate Events. 1st Edition, paperback ISBN: 9780128105047, eBook ISBN: 9780128105054, Imprint: Elsevier, 5th November 2019.

Tigges, F., Krauns, F., Hafner, A., Henze, R. (2017). Controller concept for automated lateral control. In 8th International Munich Chassis Symposium 2017, 465-481.

Trullos, J., Junghans, M., Gimm, K., Dotzauer, M. (2021). Safety critical event detection - Applying and evaluating different Surrogate Safety Measures in a roundabout traffic scenario. Accepted as poster presentation at 33th ICTCT conference, 28.-29. October 2021, Berlin, Germany. 
Urmson, C., Baker, C., Dolan, J., Rybski, P., Salesky, B., Whittaker, W., Ferguson, D., Darms, M. (2009). Autonomous Driving in Traffc: Boss and the Urban Challenge. In AI Magazine 30 (2009), No. 2, pp. 17-28

Vlakveld, W., van der Kint, S., Hagenzieker, M. P. (2020). Cyclists' intentions to yield for automated cars at intersections when they have right of way: Results of an experiment using highquality video animations. Transportation Research Part F: Traffic Psychology and Behaviour, Volume 71, May 2020, 288-307. doi: 10.1016/j.trf.2020.04.012.

Wang, S., Li, Z. (2019). Exploring the mechanism of crashes with automated vehicles using statistical modeling approaches. In PLoS One. 2019; 14(3): e0214550. Retrieved from https://www. ncbi.nlm.nih.gov/pmc/articles/PMC6438496/, doi:10.1371/ journal.pone.0214550.

Wakabayashi D. (2018, March 19). Self-Driving Uber Car Kills Pedestrian in Arizona, Where Robots Roam. 2018. Retrieved from: https://www.nytimes.com/2018/03/19/technology/ uber-driverless-fatality.html.

Wille, J. M., Saust, F., Maurer, M. (2010). Stadtpilot: Driving autonomously on Braunschweig's inner ring road. In 2010 IEEE Intelligent Vehicles Symposium. Piscataway, NJ, USA 506-511.

Zhan, W., Liu, C., Chan, C.-Y., Tomizuka, M. (2016). A nonconservatively defensive strategy for urban autonomous driving. In Proc. IEEE 19th Int. Conf. Intell. Transp. Syst., Nov. 2016, pp. 459-464, doi: 10.1109/ITSC.2016.7795595.

Zhou, H., Ivan, J. N., Gårder, P. E., Ravishanker, N. (2017). Gap acceptance for left turns from the major road at unsignalized intersections. Transport, 32(3), 252-261. doi:10.3846/16484142. 2014.933445.

Ziegler, J., Dang, T., Franke, U., Lategahn, H., Bender, P., Schreiber, ... Zeeb, E. (2014). Making Bertha Drive - An Autonomous Journey on a Historic Route. In IEEE Intelligent Transportation Systems Magazine 6 (2014), No. 2, 8-20. 